\title{
Controlling Cavitation-Based Image Contrast in Focused Ultrasound Histotripsy Surgery
}

\author{
Steven P. Allen, ${ }^{1 *}$ Timothy L. Hall, ${ }^{1}$ Charles A. Cain, ${ }^{1}$ and Luis Hernandez-Garcia ${ }^{2}$
}

Purpose: To develop MRI feedback for cavitation-based, focused ultrasound, tissue erosion surgery (histotripsy), we investigate image contrast generated by transient cavitation events.

Methods: Changes in GRE image intensity are observed while balanced pairs of field gradients are varied in the presence of an acoustically driven cavitation event. The amplitude of the acoustic pulse and the timing between a cavitation event and the start of these gradient waveforms are also varied. The magnitudes and phases of the cavitation site are compared with those of control images. An echo-planar sequence is used to evaluate histotripsy lesions in ex vivo tissue.

Results: Cavitation events in water cause localized attenuation when acoustic pulses exceed a pressure threshold. Attenuation increases with increasing gradient amplitude and gradient lobe separation times and is isotropic with gradient direction. This attenuation also depends upon the relative timing between the cavitation event and the start of the balanced gradients. These factors can be used to control the appearance of attenuation while imaging ex vivo tissue.

Conclusion: By controlling the timing between cavitation events and the imaging gradients, MR images can be made alternately sensitive or insensitive to cavitation. During therapy, these images can be used to isolate contrast generated by cavitation. Magn Reson Med 73:204-213, 2015. (c) 2014 Wiley Periodicals, Inc.

Key words: histotripsy; cavitation; focused ultrasound surgery; tissue erosion; tissue homogenization; intravoxel incoherent motion

\section{INTRODUCTION}

"Histotripsy" is a therapeutic ultrasound technique that uses focused acoustic pulses to break apart soft tissue (1-3). As these pulses arrive at the focus, they trigger the spontaneous generation of a dense cloud of bubbles, which then expands and collapses over the course of tens to hundreds of microseconds $(4,5)$. Repeated cavitation events in soft tissue transforms otherwise intact cellular structures into a highly homogenous, acellular slurry $(2,3,6)$.

MRI is a candidate for noninvasive feedback of histotripsy therapy. Ex vivo samples of porcine liver and

${ }^{1}$ Department of Biomedical Engineering, University of Michigan, Ann Arbor, Michigan, USA.

${ }^{2} \mathrm{fMRI}$ Laboratory, Department of Biomedical Engineering, University of Michigan, Ann Arbor, Michigan, USA.

${ }^{*}$ Correspondence to: Steven P. Allen, M.S., Department of Biomedical Engineering, University of Michigan, 2131 Gerstacker, 2200 Bonisteel, Blvd., Ann Arbor, MI 48109-2099. E-mail: stevepa@umich.edu

Received 6 November 2013; revised 12 December 2013; accepted 13 December 2013

DOI 10.1002/mrm.25115

Published online 27 January 2014 in Wiley Online Library (wileyonlinelibrary. com).

(c) 2014 Wiley Periodicals, Inc. canine prostate have shown increased $\mathrm{T}_{1}, \mathrm{~T}_{2}$, and diffusion weighted contrast in response to therapy (7-9). Using fast-imaging methods, MRI may provide real-time observations of the target during therapy.

Previous work has shown that cavitating micro-bubbles can cause localized signal attenuation in gradient echo (10) and spin echo (11) images. It is possible that these effects will interfere with MR-guided therapy. For example, signal attenuation caused by the cavitation cloud may mask $\mathrm{T}_{2}$ enhancement generated by tissue homogenization. Conversely, this signal attenuation may be useful for targeting or cavitation verification, and thus be a desirable part of the image. Controlling a sequence's sensitivity to cavitation can help in both these situations.

The use of microbubbles in MRI has been an area of active research. For example, microbubbles have been studied as a potential susceptibility contrast agent (12-16). They have also been used in focused ultrasound therapies to temporarily open the blood brain barrier (17-19). In both of these applications, the microbubbles involved are made of lipid or protein shells and are injected into the subject's vasculature. In contrast, the study of MR guided histotripsy is unique because the microbubbles involved are thought to originate from small gas nuclei that are native to the target tissue and have much shorter lifetimes than their manufactured counterparts.

Our general hypothesis is that cavitation causes intravoxel incoherent motion (IVIM) of water molecules. Pulse sequences with high sensitivity to IVIM will show strong contrast at the therapy site, whereas pulse sequences with reduced sensitivity to IVIM will only show the underlying tissue. A combination of these images should provide a precise image of the cavitation process.

\section{Histotripsy}

During histotripsy therapy, microbubbles are generated when an incoming ultrasound pulse with a large peak negative pressure impinges upon small gas nuclei present in the target $(4,5)$. These nuclei expand over just a few microseconds into a dense cloud of bubbles, which then collapses over the course of hundreds of microseconds (20,21). After collapse, a distribution of bubbles, with diameters on the order of $5 \mu \mathrm{m}$, remain present in the fluid for up to several seconds before dissolving (22). In unpublished work, using high-speed camera imaging, we have estimated this residual cloud to have a volume fraction well below $1 \%$.

\section{Flow at Cavitation}

The collapse phase of the cavitation cycle begins when water rushes in at high velocity toward the center of the bubble cloud. During this process, the bubble cloud 
fragments into many smaller bubbles. The flow patterns within the cavitation region become very complex and can no longer be described as conducted along radial lines toward the center of the cloud. Instead, when observed with high-speed camera imaging, these patterns are incoherent in nature, occur at very high speeds, and rarely repeat from cavitation event to cavitation event. After collapse, the flow patterns gradually return to those of thermal diffusion.

\section{IVIM Imaging}

In intravoxel incoherent motion (IVIM) imaging, the magnitude of the MR signal is attenuated if the water molecules within a voxel travel along random or pseudo-random trajectories while in the presence of balanced field gradients. The technique is an extension of the pulsed-gradient method for diffusion coefficient measurement (23).

Standard pulse sequences such as gradient recalled echo (GRE) and spin-echo sequences can be converted into an IVIM sequence by inserting a bipolar gradient pulse between the radiofrequency (RF) excitation pulse and signal acquisition (24). In the presence of these gradient lobes, random motions of water molecules cause a random distribution of phase across a voxel. When these phases are summed together, they add deconstructively. In an IVIM image, regions of enhanced random motion exhibit reduced signal intensity when compared with regions with restricted random motion.

It has also been demonstrated that the flow of the water molecules need not be entirely incoherent to observe IVIM contrast, because coherent flow distributed across several velocities will also produce signal attenuation (24). However, the coherent motion will also introduce some net gain of phase to a voxel. Changes in image phase due to coherent motion play an integral role behind shear-wave (25-27), acoustic radiation force $(28,29)$, and acoustic streaming imaging (30).

During an IVIM imaging experiment, the signal observed at the echo time (TE) can be expressed by

$$
A=F A_{0}
$$

where $A_{0}$ is the magnitude of the MR signal observed at TE if the encoding gradients are disabled. The attenuation factor, $F$, depends on both the motions of the water molecules and the encoding gradient waveforms and is also subject to the constraint $F \leq 1$.

A simple model for IVIM imaging consists of a voxel where populations of water undergo different forms of isotropic movement. Molecular exchange between populations occurs more slowly than the duration of the imaging sequence. In this case, $F$ can be calculated using the equation

$$
F=\sum_{i} f_{i} e^{-b D_{i}}
$$

where $f_{i}$ is the fraction of water molecules in the ith population and $\sum f_{i}=1$. The term $D_{i}$ is the apparent diffusion coefficient of the ith population. The term $b$ takes the form

$$
b=\gamma^{2} \int_{0}^{T E}\left[\int_{0}^{t} G(\tau) d \tau\right]^{2} d t
$$

where $\gamma$ is the gyromagnetic ratio and $G(\tau)$ is the timedependent vector of the gradient pulses used in the imaging experiment (31).

The work in (32) has shown that contributions to signal attenuation from populations undergoing some sort of nonthermal motion are separable from the contributions caused by those undergoing thermal diffusion. This is because the populations of water undergoing the nonthermal motion often have a larger apparent diffusion coefficient than the populations undergoing solely thermal diffusion. Gradient waveforms can be found with values for $b$ such that the majority of signal attenuation is caused by only the nonthermal motion. It may be possible to leverage this concept to isolate contrast generated by cavitation.

\section{Histotripsy and IVIM Imaging}

The collapse phase of the histotripsy therapy cloud may cause sufficient incoherent motion of the water molecules that the MR signal will be attenuated in an IVIM image. This attenuation may be separable from attenuation caused by diffusion and perfusion by using motionencoding gradient waveforms with small values for $b$.

Furthermore, the incoherent motions of water molecules induced by cavitation may be different from the motions that characterize thermal diffusion. Therefore, IVIM images of a cavitation event with gradient waveforms that have identical $b$ values but vary in shape and amplitude may yield different signal attenuation factors.

IVIM at the therapy site may also be caused by the mechanisms of acoustic streaming and acoustic radiation forces. Their contributions to IVIM may be separated from the contribution made by cavitation by repeatedly imaging the cavitation site while increasing the amplitude of the acoustic pulses. The signal attenuation caused by streaming and radiation forces should continuously increase with pulse amplitude. The signal attenuation caused by cavitation should only be observable when the peak negative pressure of the acoustic pulse exceeds the cavitational threshold pressure.

If, during an IVIM sequence, the motions of the water molecules have already returned to thermal equilibrium by the start of the first encoding gradient, the resulting image may not capture signal contrast specific to cavitation. Instead, it will only capture attenuation caused by other incoherent processes. It may be possible, by controlling the relative timing between a cavitation event and the IVIM gradients, to control the appearance of signal attenuation on the resulting image.

In the following, we investigate the dependence of the attenuation factor on the peak negative pressure of the acoustic pulses as well as the amplitudes, separation times, and direction of the IVIM gradient pairs. We also investigate the dependence of the attenuation factor on the timing between a cavitation event and the start of the IVIM gradients. We do so either by inserting bipolar gradient lobes into a standard GRE spin-warp sequence one direction at a time, or by placing the cavitation events some time before or after the spin-warp sequence's 
imaging gradients (which also act as IVIM-encoding gradients). We then construct an imaging sequence to alternately enhance or minimize IVIM attenuation during MR-guided therapy of an ex vivo liver sample.

We find that signal attenuation in water is observed only when the peak negative pressures of the acoustic pulses exceed $24 \mathrm{MPa}$ and that the magnitude of attenuation depends upon the amplitudes and separation times of the IVIM-encoding gradients. The magnitude of attenuation is found to be isotropic with IVIM encoding direction and varies strongly with the relative timing between the cavitation event and a bipolar IVIM-encoding gradient waveform. Pulse sequences with b-values on the order of $0.04 \mathrm{~s} / \mathrm{mm}^{2}$ have sufficient sensitivity to IVIM for cavitation to induce greater than $10 \%$ signal reduction. In the ex vivo tissue sample, images with varied sensitivity to cavitation can be combined to form a precise image of the cavitation site.

\section{METHODS}

All experiments were conducted using an MRcompatible focused transducer attached to a water bath (Fig. 1). Experiments A-D were performed in tap water and experiment $\mathrm{E}$ was performed in excised porcine liver. The details of the ultrasound therapy and MR imaging are given below.

\section{Cavitation Generation}

Cavitation was stimulated using an MR-safe, spherically focused array of 7 circular, ceramic, piezoelectric elements with a diameter of $2 \mathrm{~cm}$ each and a resonant frequency of $1 \mathrm{MHz}$. The elements were arranged confocally along the portion of a spherical surface with a $4.5-\mathrm{cm}$ radius that is subtended by 4.8 steradians. The overall circular aperture of the transducer is $8.5 \mathrm{~cm}$ (see Fig. 1A).

The transducer was connected to an amplifier constructed by our lab that is able to deliver high-voltage, short duration pulses to the transducer elements. The amplifier was controlled by a programmable trigger pulse from the MR system console. Once triggered by the console, the amplifier and transducer setup launched a single acoustic ultrasound pulse, which arrived at the transducer focus approximately $28 \mu$ s later. Increasing the voltage across the amplifier resulted in increasing the peak negative pressure of the acoustic waveform.

Two water baths were constructed to keep the ultrasound beam path immersed. Each water bath had a

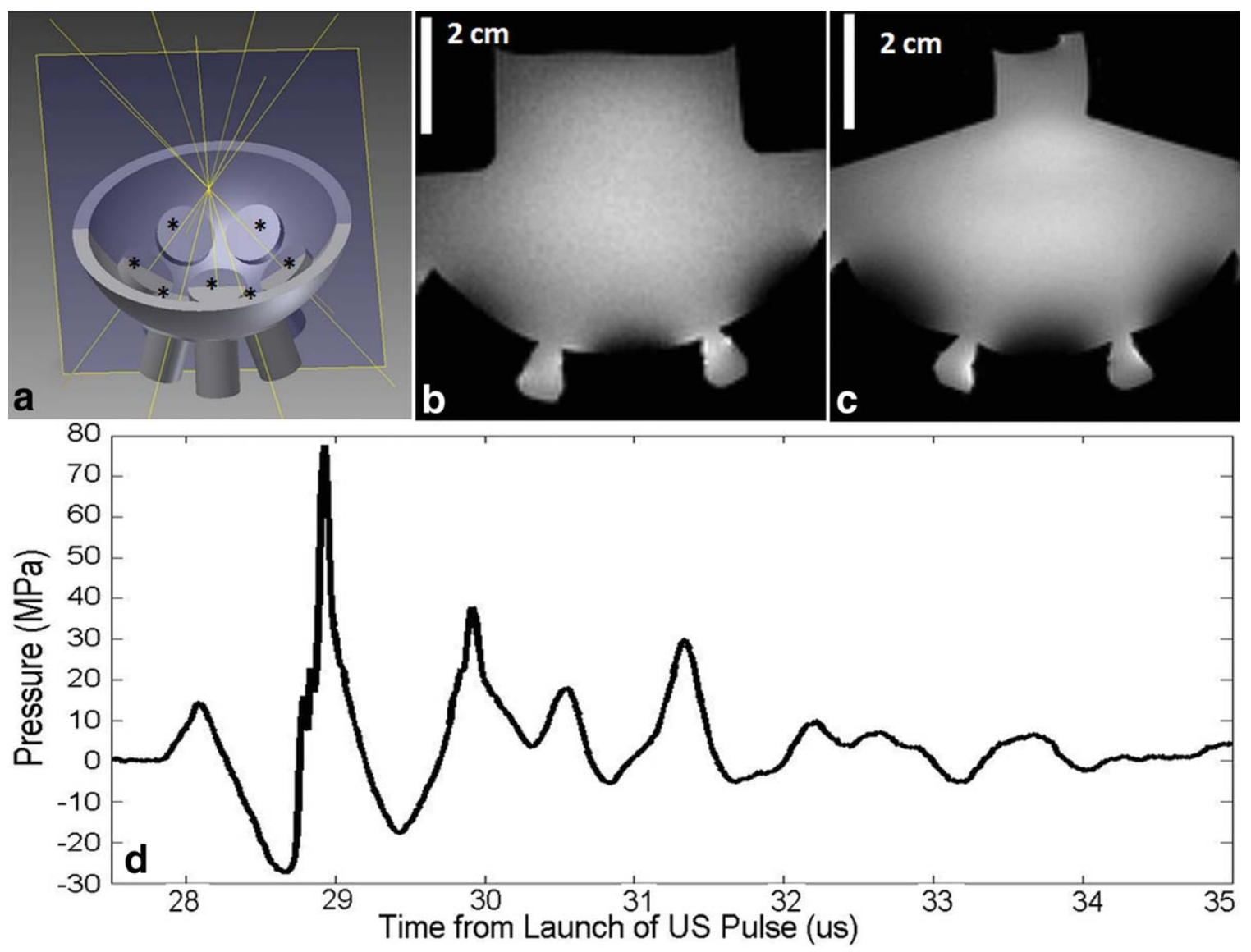

FIG. 1. Experimental setup. A: The acoustic pulses emitted from the piezoelectric elements $\left(^{\star}\right)$ converge at the focus (yellow lines). The imaging plane transects the focus and the transducer (blue surface). Two different water baths can be attached to the transducer to keep the beam path submerged in water. A spin-echo image of the larger water bath is shown in (B). The smaller-necked water bath is shown in (C). A trigger pulse can be sent from the MR pulse controller to the US amplifier at various times during an imaging sequence as chosen by the operator. D: The acoustic waveform at the focus generated with maximum driving voltage as estimated by summing together the outputs of the individual elements. 
different size neck to support different transmit/receive coils. The water bath was attached to the transducer housing in such a way that the transducer could be placed on the table of the MR scanner with the transmitting surface facing up. Once attached, the water-bath volume was filled with tap water (see Fig. 1B,C).

The acoustic output at the transducer focus was estimated using a fiber-optic hydrophone (33). Because cavitation disrupts the accuracy of the hydrophone, the amplifier excited each transducer element individually with incrementing driving voltage. The combined acoustic output of the transducer was then estimated by summing together the acoustic outputs of the individual elements. The estimated combined output of the transducer at the maximum driving voltage is shown in Figure 1D and has a peak negative pressure of 27.5 MPa. This driving voltage was subsequently used for all experiments where cavitation was desired.

Before the start of the experiments, cavitation was confirmed to occur in both water and tissue using an ultrasound imaging probe. With the voltage across the amplifier set to maximum, trigger pulses sent to the amplifier caused a temporally variable, hyperechoic region in the ultrasound images. Cavitation was assumed to continue to occur when ultrasound probe was removed and the water or tissue sample was placed inside the scanner.

For control experiments, where cavitation was not desired, the amplifier voltage was reduced by half and the estimated peak negative pressure of the resulting acoustic pulse was 15.2 MPa. At these pressures, a hyperechoic region on the ultrasound images was never observed for every trigger pulse sent to the amplifier.

\section{MR Images}

Unless otherwise stated, all IVIM images were acquired on a 7T/310 small animal scanner (Agilent Technologies, Walnut Creek, CA) using a 14-cm diameter, dualchannel, birdcage coil. The following parameters were used: echo time/repetition time $(\mathrm{TE} / \mathrm{TR})=10 / 2500 \mathrm{~ms}$, field of view $(\mathrm{FOV})=55 \times 55 \times 1 \mathrm{~mm}$, matrix $=128 \times$ $128 \times 1$, bandwidth $=50 \mathrm{kHz}$, flip angle $=90^{\circ}$, number of excitations $(\mathrm{NEX})=1$. Images were acquired in the axial plane with the phase encode direction displayed as the up/down direction of the image. The therapy transducer was pulsed only once per TR. Thus, a 128-shot GRE imaging sequence delivered 128 ultrasound pulses to the target over the duration of the sequence. Long repetition times were used decouple any effects caused by concatenated pulses and reduce through-plane motion effects.

\section{Experiment A: Dependence on Ultrasound Pulse Amplitude}

An experiment was performed to determine the dependence of IVIM based signal attenuation on the amplitude of the ultrasound pulse. Tap water at the focus of the transducer was repeatedly imaged by identical IVIM sequences while the voltage across the transducer amplifier was incremented. The voltage increments corresponded to peak negative pressures of 9.0, 11.0, 14.0, 15.1, 16.3, 18.8, 21.3, 23.4, 24.6, 24.5, 25.2, 25.8, and 27.5 MPa. A control image was also acquired with the amplifier turned off.
The IVIM sequence consisted of a GRE spin warp sequence with a balanced, bipolar gradient pair inserted between RF excitation and the readout gradient and oriented along the slice-select direction. Immediately after completion of the first IVIM gradient lobe, a trigger pulse was sent from the MR console to the transducer amplifier. The duration of each IVIM gradient lobe was $1 \mathrm{~ms}$. The amplitude of the IVIM gradient was $3.5 \mathrm{Gcm}^{-1}$ and the separation time between the two lobes was $3.5 \mathrm{~ms}$. Images were acquired with the smaller-necked cap and a 1.5-cm-diameter surface coil centered about the cavitation site. FOV $=30 \times 30 \times 1 \mathrm{~mm}$ (see Fig. $2 \mathrm{~A}$ ).

\section{Experiment B: Dependence on the IVIM Gradient Waveform}

Two experiments were conducted to determine how signal attenuation at the cavitation site varies with the amplitudes and mixing time of an IVIM-encoding gradient waveform. For both experiments, a balanced, bipolar gradient pair was inserted between the RF excitation and readout portions of a GRE spin warp sequence. These IVIM gradients were oriented along the slice select direction. Immediately after completion of the first IVIM gradient lobe, a trigger pulse was sent from the MR console to the transducer amplifier. The duration of each IVIM gradient lobe was $1 \mathrm{~ms}$.

The first experiment acquired eight images of tap water at the transducer focus with the IVIM-encoding gradient amplitudes set to $3.4 \mathrm{Gcm}^{-1}$. The separation between the two IVIM gradient lobes was varied from 0 to $3.5 \mathrm{~ms}$ in $0.5 \mathrm{~ms}$ increments (see Fig. 2A).

The second experiment acquired 21 images with the IVIM-encoding gradient amplitudes varying from 0 to 9.5 $\mathrm{Gcm}^{-1}$ in $0.47 \mathrm{Gcm}^{-1}$ increments. The separation between the IVIM gradient pulses was kept to $10 \mu \mathrm{s}$ for all instances-just long enough to transmit a trigger pulse from the MR console to the ultrasound amplifier. (see Fig. 2B).

\section{Experiment C: Directional Dependence}

The dependence of the attenuation factor on gradient direction was explored. Here, the same sequence used in both experiment $\mathrm{A}$ and the first part of experiment B was repeated 3 times with the IVIM-encoding gradients directed alternately along the slice-select, readout, and phase-encode directions. For each run, a total of eight images of tap water at the transducer focus were acquired with the IVIM-encoding gradient amplitude set to 3.5 $\mathrm{Gcm}^{-1}$ while the mixing time was varied from 0 to $3.5 \mathrm{~ms}$ in 0.5-ms increments. A trigger pulse was sent from the MR console to the transducer amplifier immediately after completion of the first IVIM encoding lobe (see Fig. 2A).

Imaging was carried out with a 1.5-cm-diameter surface coil centered about the cavitation site to boost the signal to noise ratio. Imaging parameters were $\mathrm{FOV}=30$ $\times 30 \times 1 \mathrm{~mm}$, Matrix $=64 \times 64 \times 1$.

\section{Experiment D: Dependence on Timing Relative to IVIM- Encoding Gradients}

To explore how the signal attenuation at the cavitation site depends upon the relative timing between a cavitation event and the imaging gradients, GRE spin warp images of tap water at the transducer focus were 


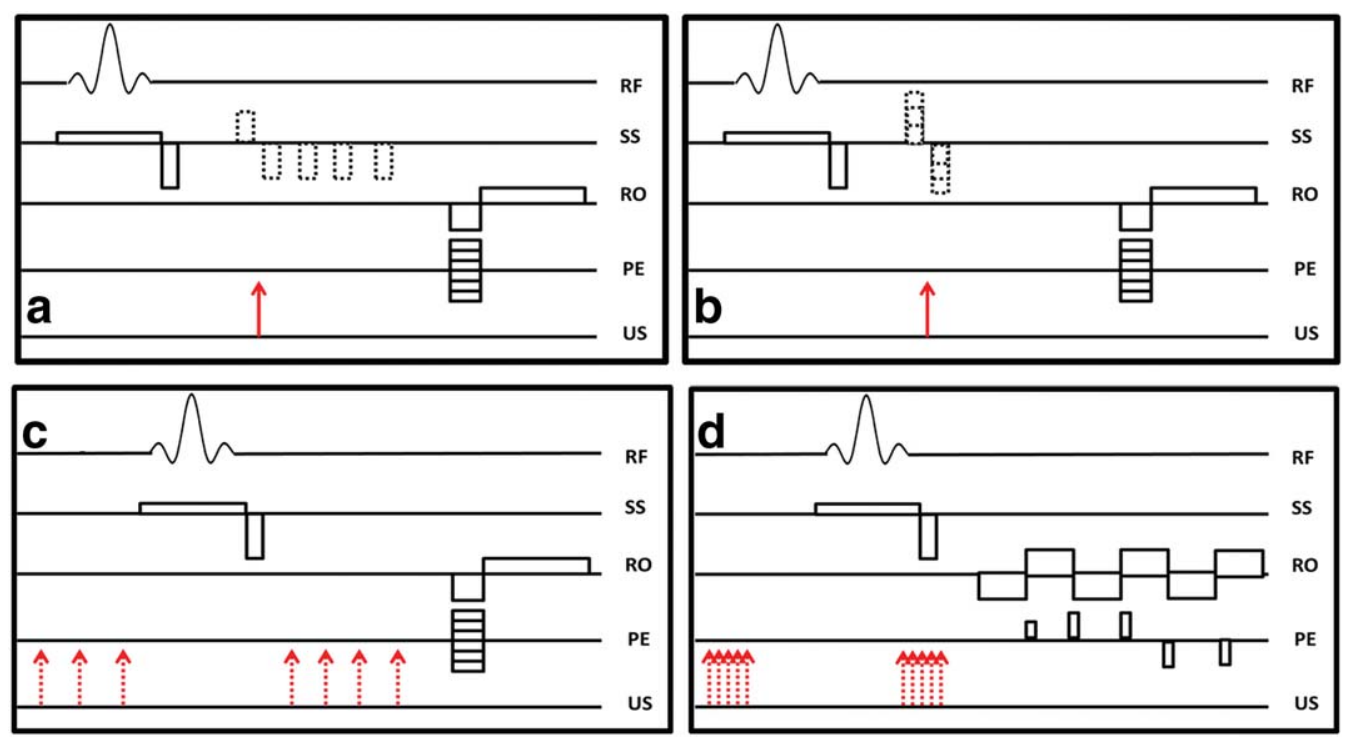

FIG. 2. Schematic of pulse sequences used: (A) GRE, spin-warp, IVIM sequence used in experiments A, B1, and C. The cavitation event occurs just after the first IVIM gradient lobe (red arrow) while separation time is incremented. B: GRE, spin-warp, IVIM sequence used in experiment B2. The separation time between the IVIM gradients is held constant while the amplitude is incremented. C: GRE, spinwarp pulse sequence used in experiment D. The ultrasound pulse is fired at different times before signal acquisition (red arrows). D: GRE EPI sequence used in experiment E. Ultrasound pulses are either fired $10 \mathrm{~ms}$ before RF acquisition to desensitize the sequence to cavitation IVIM, or fired between the slice-select and slice-rewind gradient lobes to sensitize the sequence to cavitation IVIM. Each instance/increment is shown as a dotted line for clarity. Amplitudes and time gaps are not to scale. Abbreviations are: RF $=$ radio frequency, $\mathrm{SS}=$ slice select, $\mathrm{RO}=$ readout, $\mathrm{PE}=$ phase encode, $\mathrm{US}=$ ultrasound.

acquired while placing the cavitation event at different times relative to the RF pulse (see Fig. 2C).

The trigger timings used were composed of twelve 1-ms increments ranging from 11.1 to $0.1 \mathrm{~ms}$ before the center of the RF excitation pulse, and twelve 0.5-ms increments ranging from 2.3 to $7.8 \mathrm{~ms}$ after the center of the RF excitation pulse. Gradient parameters are: slice-select/rewind amplitude $=3.2 / 4.0 \mathrm{Gcm}^{-1}$; slice select/rewind duration = 2.1/0.9 ms; readout/rewind amplitude $=2.1 / 4.0 \mathrm{Gcm}^{-1}$; readout/rewind duration $=2.6 / 0.8 \mathrm{~ms}$. The slice-select and readout gradient pairs had b-values of 0.06 and 0.05 $\mathrm{smm}^{-2}$, respectively.

\section{Experiment E: Ex Vivo Tissue Sample}

The ability to control cavitation induced IVIM contrast in a therapy setting was examined by simultaneously scanning and treating an excised tissue sample. To demonstrate that fast imaging sequences can be used to detect cavitation-based IVIM contrast, a GR-EPI sequence was used instead of a spin-warp sequence.

A porcine liver sample was suspended in the smaller water bath. The 1.5-cm-diameter surface coil was placed around the water bath neck and centered about the cavitation site. After applying 5000 ultrasound pulses to the liver tissue, the therapy site was scanned twice by the EPI sequence. During scanning, 10 cavitation pulses were triggered by the scanner console. These pulses were placed either $10 \mathrm{~ms}$ before the start of the sliceselect gradient, or between the slice-select and slicerewind gradient lobes (see Fig. 2D).
Each trigger pulse was spaced apart by $0.2 \mathrm{~ms}$. The sequence imaged the same plane as experiments A-D. Imaging parameters: TE/TR: 7/1000 ms, FOV $=33 \times 33 \times$ $1 \mathrm{~mm}$, matrix $=96 \times 96 \times 1$, bandwidth $=208 \mathrm{kHz}$, flip angle $=70^{\circ}, \mathrm{NEX}=1$, Segments $=12$. A reference echo for each segment was acquired for each image. This made for a total of 24 shots and 24 ultrasound pulses per image.

\section{Control Images}

The above experiments were repeated with the voltage across the ultrasound amplifier turned down to below the cavitation threshold (estimated peak negative pressure: 15.2 MPa).

\section{Data Processing}

The attenuation factor, $F$, of each image was estimated by selecting a region of interest (ROI) within the area of attenuated signal and averaging the intensity of the pixels within this ROI. This result was then divided by the average signal intensity of the pixels within the same ROI when applied to the corresponding control image. An example ROI is shown in Figure 3.

\section{RESULTS}

An example of images of the transducer, water bath, cavitation-induced artifacts, and ROI selection are shown in Figure 3. The image shown in Figure 3B indicates a signal void created by the presence of a cavitation bubble (thick solid arrow), has an attenuation factor of 0.25 , and 

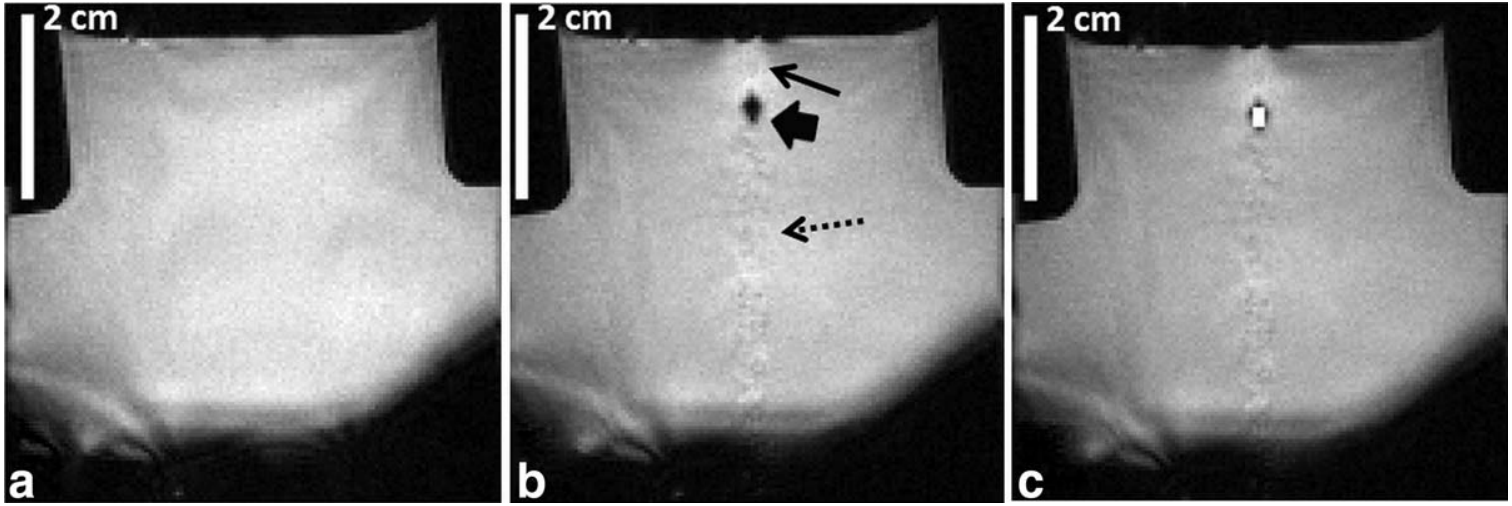

FIG. 3. A: Gradient echo image acquired with the sequence shown in Figure $2 \mathrm{~A}$ and with the voltage across the US amplifier set below the cavitation threshold. B: Image acquired by the same sequence but with the voltage across the US amplifier set above the cavitation threshold. Attenuation factor: 0.25 ; bvalue: $0.47 \mathrm{smm}^{-2}$. The cavitation event causes localized attenuation of signal magnitude (block arrow). Additionally, the cavitation event causes signal enhancement due to through-plane motion (solid arrow) as well as ghosting (dotted arrow) along the phase-encode direction (up/down). C: The same image as in (B) but with the ROI used for calculations highlighted as bright pixels.

is taken from the first part of experiment $\mathrm{B}$ with a gradient separation time of $3.5 \mathrm{~ms}$.

The image in Figure 3B also displays ghosting along the phase encoding direction (up/down) and some signal enhancement near the attenuation site (dotted and narrow, solid arrows, respectively). We observed that these artifacts remained consistent across all imaging experiments where the voltage across the ultrasound amplifier was above the cavitation threshold. The ghosting artifact is caused by random variations in the shape and evolution of the bubble cloud between shots. The signal enhancement artifact is thought to be caused by throughplane motion of the water molecules between shots. Its intensity was found to reduce with both increased slice thickness and increased TR. These artifacts were also not visible in any of the control images.

An example ROI used to calculate the attenuation factor is shown in Figure 3C.

\section{A: Dependence on Ultrasound Pulse Amplitude}

The attenuation factors estimated while varying the amplitude of the ultrasound pulse are plotted as a function of peak negative pressure in Figure 4. As the negative pressure phase of the ultrasound pulse increases in magnitude, the attenuation factor remains near unity until $24 \mathrm{MPa}$, where it quickly decreases with increasing peak negative pressure of the acoustic pulse.

\section{B: Dependence on the IVIM Waveform}

The estimated attenuation factors measured from the experiments changing the IVIM gradient amplitudes and mixing time are plotted as a function of b-value in Figure $5 \mathrm{~A}$.

As shown in the figure, the amount of attenuation increases as the sequence is further sensitized to incoherent motion. However, the variation of the attenuation factor depends upon how the IVIM gradients were altered. Changes in the mixing time seem to cause a greater effect on the attenuation factor than changes in the gradient amplitudes. Both curves seem to follow an exponential decay pattern typical of pseudo-random flow in IVIM imaging, but with different decay rates. The jumps in the decay curve for the gradient amplitude varying experiment correspond to images with intrashot motion artifact.

\section{C: Dependence on Direction}

The estimated attenuation factors measured in response to different directions of the encoding gradients are displayed in Figure 5B. As in the previous experiment, the attenuation factor decreases monotonically with increased $b$ value of the gradient waveforms. Furthermore, the signal attenuation has an isotropic response to gradient direction.

Example magnitude and phase images of the directionality experiment are displayed in Figure 6. These images were acquired with the IVIM gradients at maximum sensitivity to motion ( $3.5 \mathrm{~ms}$ separation time, $0.53 \mathrm{smm}^{-2} \mathrm{~b}$ value) and generated along the phase encode direction (up/down). The IVIM motion causes noticeable

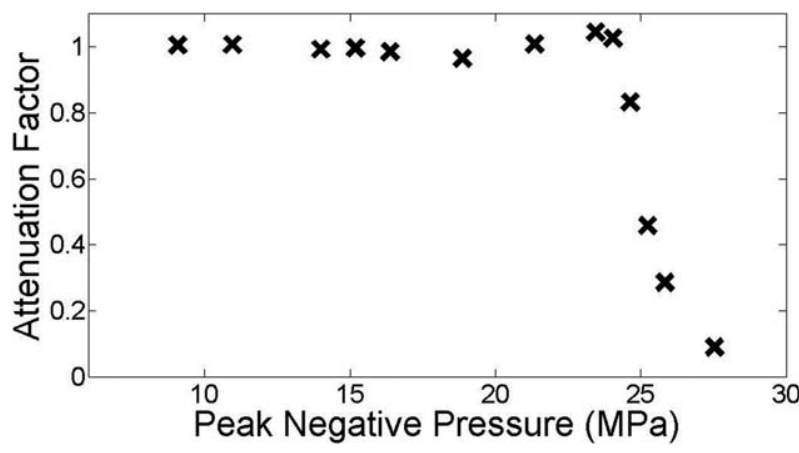

FIG. 4. Results corresponding to experiment A. The attenuation factors found at the cavitation site for various applied acoustic pulses are plotted versus the peak negative pressure of those acoustic pulses. The attenuation factor remains near unity until the peak negative pressure of the ultrasound pulse approaches 24 $\mathrm{MPa}$, where it decreases dramatically with increased peak negative pressure. This threshold pressure matches up with previous studies of cavitation in unfiltered tap water. 

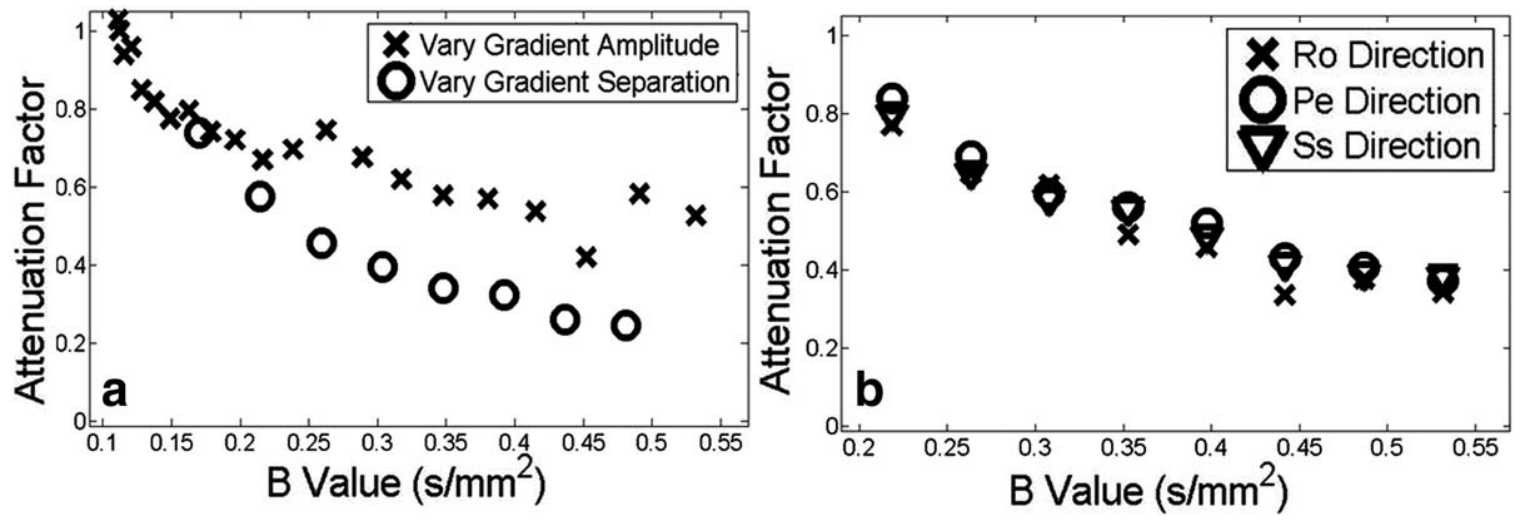

FIG. 5. Results corresponding to experiments B,C. A: The attenuation factor at the cavitation site estimated from experiment B is plotted versus the $b$ value of the IVIM-encoding gradients. MR signal attenuation is greater when motion is sensitized by separating the gradient lobes $(X)$ than by increasing the gradient strength $(O)$ even for experiments with equivalent $b$-values. B: The attenuation factors found at the cavitation site for experiment $C$ are plotted versus the $b$ value of the IVIM-encoding gradients. The IVIM-encoding gradients were played along the readout $(X)$, phase-encode $(\mathrm{O})$, and slice-select $(\Delta)$ directions. The $b$ value was increased by incrementing the mixing time between IVIM gradient lobes. The behavior of the attenuation factor is isotropic with gradient direction. Note that these b-values are extremely small, which suggests a very large amount of IVIM compared with thermal diffusion and tissue perfusion processes.

attenuation at the focal zone (attenuation factor $=0.32$ ) and a opposite changes in phase localized around the cavitation site. A large amount of phase also accrues at the top of the object due to an air bubble.

\section{D: Dependence on Cavitation and IVIM Gradient Timing}

The attenuation factors estimated while varying the timing of the trigger pulses are plotted in Figure 7. In this figure, time relative to the center of the RF pulse (sinc shape) is plotted along the $x$-axis while the attenuation factor is plotted along the $y$-axis. Each asterisk marks the attenuation factor observed when an ultrasound pulse is programmed to occur at the corresponding time on the $x$-axis.

As shown in the figure, the estimated attenuation factor decreases as the cavitation event approaches either the slice select or readout gradient pairs. Furthermore, cavitation events that occur immediately after the slice select pair do not display appreciable signal attenuation. The slice select and readout gradients appear to cause equal amounts of signal attenuation.

\section{E: Ex Vivo Tissue Sample}

The separability of cavitation based IVIM contrast is demonstrated in Figure 8. High-resolution GRE EPI images of the liver sample are shown in frames (A,B). In frame (A), 10 ultrasound pulses are applied $10 \mathrm{~ms}$ before the start of the slice-select gradient. In frame (B), 10 ultrasound pulses are applied between the slice-select and slice-rewind gradients. The region of $\mathrm{T}_{2}{ }^{*}$ enhanced contrast indicated by the arrow in frame (A) specifies homogenized liver tissue. The localized signal attenuation indicated by the arrow in frame (B) specifies cavitationinduced IVIM contrast. The pixel-by-pixel subtraction of the two images is shown in Figure 8C. In this manner, cavitation induced contrast can be isolated from other image features.

\section{DISCUSSION}

\section{Cavitation}

The results shown in Figure 4 corroborate well with cavitation theory. In the figure, the attenuation factor decreases dramatically when the peak negative pressure of the acoustic pulse transitions from 24.6 to $27.5 \mathrm{MPa}$. Meanwhile, reference 4 reports that the probability of 5$\mu s$ acoustic pulses in unfiltered water to successfully induce cavitation increases from 0 to 1 as the peak negative pressure of each pulse increases through a nearly identical range of 24 to $28 \mathrm{MPa}$. In contrast, acoustic
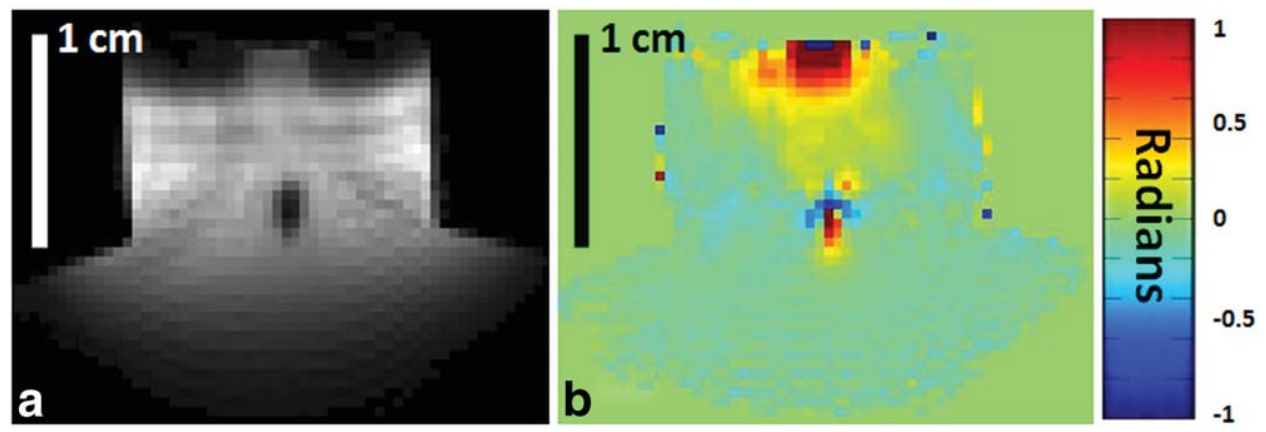

FIG. 6. Example magnitude (A) and phase (B) images from the results of experiment C. For this image, separation time $=3.5 \mathrm{~ms} ; \mathrm{b}$ value $=0.53 \mathrm{smm}^{-2}$; attenuation factor $=0.32$. The IVIM gradients are applied along the phase encode direction (up/down). Water above and below the cavitation site accrue opposite phases and may be due to coherent flow patterns induced by cavitation. 


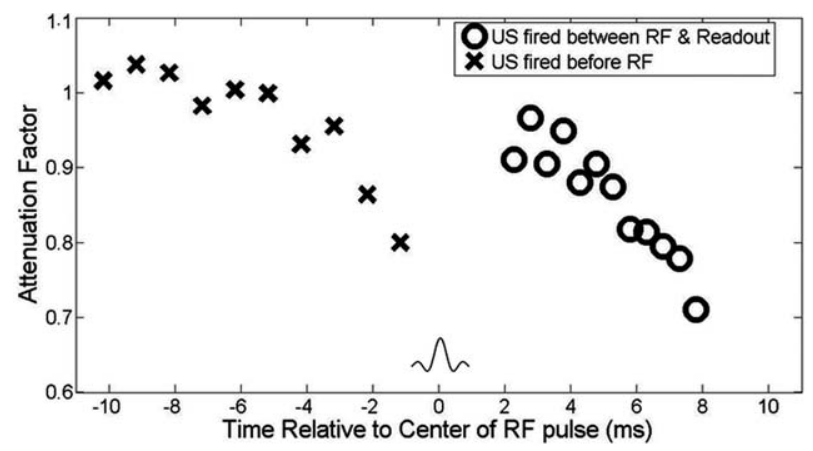

FIG. 7. Results corresponding to experiment $\mathrm{D}$. The attenuation factor is plotted as a function of the time (in ms) between the US pulse and the RF excitation pulse. The small sinc shape represents the time taken up by the RF pulse. The MR signal is most attenuated when the cavitation event occurs just before either the slice-select or the readout gradient pulses.

streaming and acoustic radiation force are reported to increase smoothly with increasing acoustic power $(28,30)$. The threshold behavior in Figure 4 , suggests that cavitation makes the primary contribution to the localized signal attenuation observed in these experiments.

\section{Effect of Susceptibility Gradients}

The sparse distributions of bubbles that linger after a cavitation event appear to have a very small effect on the attenuation factor at the cavitation site. In the literature, theoretical (14) and experimental work $(13,15,16)$ has shown that the introduction of microbubbles to water reduces the transverse relaxation rate, $\mathrm{R}_{2}{ }^{*}$, of water proportionally to the volume fraction of the bubbles. At 7 Tesla (T), this proportionality constant ranges, for $\sim 5 \mu \mathrm{m}$ diameter microbubbles, from $42.38 \mathrm{~s}^{-1}$ (16) to $58.52 \mathrm{~s}^{-1}$ (13), depending on the composition of the bubbles. In the case of histotripsy, if the bubble distribution that persists after collapse of the cavitation cloud has a volume fraction of $1 \%$, the attenuation factor in Figure 7 should display a change in $\mathrm{R}_{2}$ * decay rate of $\sim 40-50 \mathrm{~s}^{-1}$. However, such large changes in the transverse relaxation rate are not apparent in Figure 7. The attenuation factor does not decrease with increasing time separating the formation of the residual cloud and readout. Therefore, the volume fraction of this residual cloud should be much smaller than $1 \%$.

It is possible, however, that the cavitation cloud itself may be sufficient to induce some susceptibility effect on the cavitation site. In this case, due to its short duration, a cavitation event should introduce some constant signal attenuation factor to the data points in Figure 7. This constant attenuation, if present, appears to be buried in the noise.

\section{Effects of Fluid Motion}

The attenuation factor at the therapy focus correlates strongly with changes made to the IVIM-encoding gradients. Furthermore, the rate at which the attenuation factor changes depends on how the IVIM-encoding gradients are modified. Experiments where the mixing time between the gradient pairs was lengthened exhibit more signal attenuation than experiments where the amplitudes of the gradient lobes were increased. If flow induced by cavitation was similar to thermal diffusion, these attenuation factors should identical for gradients with identical $b$ values.

As shown in Figure 5B, the attenuation caused by cavitation is isotropic with gradient direction. This suggests that any gradient direction can be used to capture cavitation-based IVIM contrast.

The phase image displayed in Figure 6 shows that water on opposite sides of the cavitation site accrue opposite phases. One possible explanation for this result is that the collapse phase of the cavitation cycle occurs more slowly than the expansion phase. Water molecules may have more time to generate excess phase during the collapse portion of the cavitation cycle than during the expansion portion. If the expansion and collapse portions occurred at equal rates, then the excess phases would negate each other. In future work, phase contrast MRI may be a useful tool to study cavitation-induced flows in opaque media.
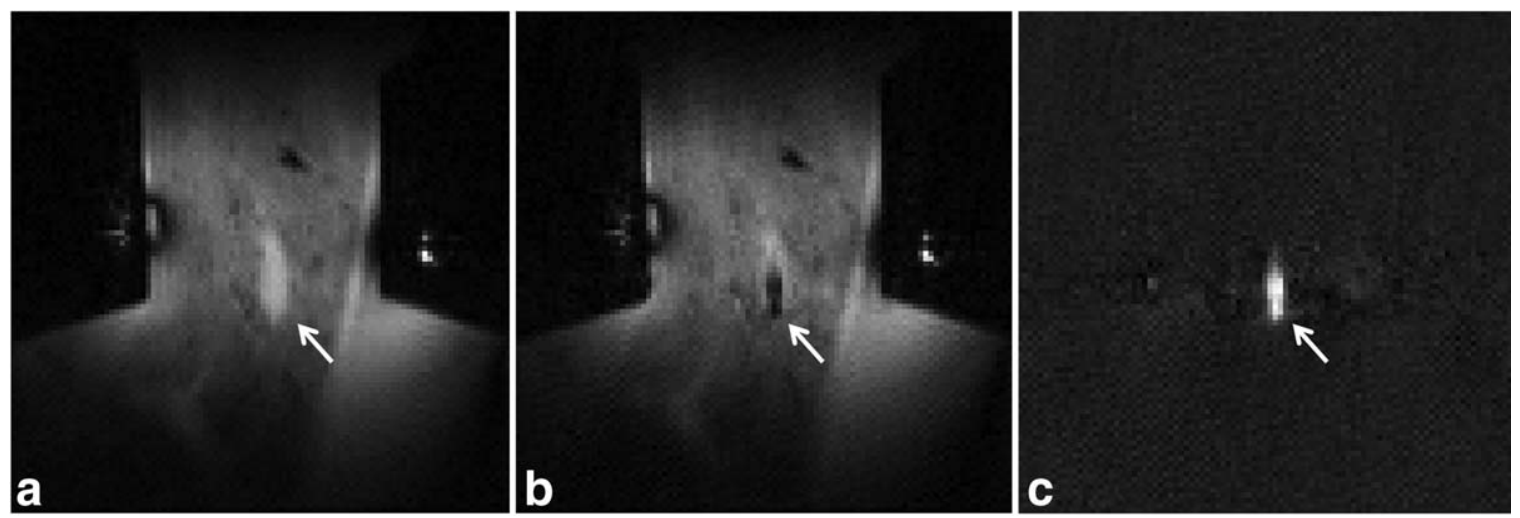

FIG. 8. Results corresponding to experiment D. EPI images acquired during therapy with (A) ultrasound pulses fired $10 \mathrm{~ms}$ before the start of the slice select gradient and $(B)$ ultrasound pulses fired between the slice-select and slice-rewind gradients. $A T_{2}{ }^{*}$ enhanced lesion is apparent in (A) at the therapy focus. Localized attenuation from a cavitation cloud is shown in (B). The pixel-wise subtraction image between $(A)$ and $(B)$ is shown in $(C)$. In this manner, the attenuation caused by cavitation can be isolated from other image features and incoherent motion processes. 
The $b$ values of the IVIM-encoding gradients used in experiments $\mathrm{A}-\mathrm{C}$ are on the same order as those of standard imaging gradient pairs. This finding corroborates the results shown in Figure 7 . In this figure, the attenuation factor decreases as the cavitation event approaches the start of either imaging gradient pair. This suggests that standard imaging gradients can sensitize an image to cavitation.

Additionally, the results in Figure 7 show that cavitation events that occur more than $5 \mathrm{~ms}$ before a gradient pair do not cause significant signal attenuation. We suspect a standard imaging sequence can be made either sensitive or insensitive to cavitation by controlling the proximity of the cavitation event to the imaging gradients.

\section{Therapy Feedback in Tissue}

The images displayed in Figure 8 demonstrate that sensitivity to cavitation can be controlled by changing the relative timing between the cavitation events and the imaging gradients. The images acquired where the ultrasound pulses are fired immediately after the slice select gradient display localized attenuation. Meanwhile, images acquired when the ultrasound pulses are fired $10 \mathrm{~ms}$ before the slice-select gradient do not display cavitation-specific contrast. A subtraction between these two images yields a precise image of the cavitation site.

Of note, more pulses (10 per TR) were needed to achieve this attenuation than needed in water (1 per TR). This is likely because the cavitation threshold in tissue is higher than in water. Maxwell et al (4) reports that the probability of cavitation in kidney begins to transition near $27 \mathrm{MPa}$.

Additionally, these images were acquired during the course of therapy ( $\sim 5000$ pulses) rather than at its onset. This is because it was difficult to obtain images with cavitation contrast at the beginning of therapy. Signal attenuation became observable only after many ultrasound pulses were applied to the tissue.

One explanation for this behavior is that, at the onset of therapy, neither cavitation nor acoustic streaming can mix about water that is bound up into intact tissue and cavitation-based contrast can only be observed in liquid or semiliquid media. A second explanation is that the cavitation cloud produced at the onset of therapy is very small due to the higher cavitation threshold in intact tissue and the relatively weak negative pressure of our transducer. A more powerful transducer should then be able to produce IVIM contrast at the onset of therapy. Currently, we are unable to either increase the peak negative pressure of the transducer or independently measure the size of the cavitation cloud during therapy while performing MR imaging. Future work that accomplishes these tasks may be able to determine which explanation is accurate.

We note that signal attenuation caused by cavitation occurs when the peak negative pressure of the acoustic pulse surpasses $24 \mathrm{MPa}$. This attenuation increases with increased sensitivity of IVIM-encoding gradient pairs. Furthermore, to capture IVIM-induced signal attenuation, the cavitation event must occur such that the water motion has not returned to thermal equilibrium by the beginning of the gradient sequence. Otherwise, the sequence will be insensitive to cavitation-based IVIM. Cavitation-based signal attenuation has an isotropic response to the direction of the IVIM-encoding gradients. Lastly, we have shown that, in an ex vivo model, sequences can be made alternately sensitive and insensitive to the attenuation caused by cavitation by merely controlling the relative timing between the cavitation event and the imaging gradients.

\section{CONCLUSIONS}

In this study, we have examined the response of the MR signal of a GRE sequence to a cavitation event. Cavitation appears to cause a temporary increase in incoherent flow of the water molecules at the cavitation site. When scanned by a sequence with a motion-sensitizing, balanced, field gradient, cavitation events exhibit localized signal attenuation in MR images. This sensitivity can be controlled by determining the placement of the cavitation events while imaging.

\section{ACKNOWLEDGMENT}

Drs. Cain and Hall have equity, royalty, and consulting interests in HistoSonics, Inc.

\section{REFERENCES}

1. Xu Z, Ludomirsky A, Eun LY, Hall TL, Tran BC, Fowlkes JB, Cain CA. Controlled ultrasound tissue erosion. IEEE Trans Ultrason Ferroelectr Freq Control 2004;51:726-736.

2. Parsons JE, Cain CA, Abrams GD, Fowlkes JB. Pulsed cavitational ultrasound therapy for controlled tissue homogenization. Ultrasound Med Biol 2006;32:115-129.

3. Roberts WW, Hall TL, Ives K, Wolf JS Jr, Fowlkes JB, Cain CA. Pulsed cavitational ultrasound: a noninvasive technology for controlled tissue ablation (histotripsy) in the rabbit kidney. J Urol 2006;175:734738.

4. Maxwell AD, Cain CA, Hall TL, Fowlkes JB, Xu Z. Probability of cavitation for single ultrasound pulses applied to tissues and tissuemimicking materials. Ultrasound Med Biol 2013;39:449-465.

5. Maxwell AD, Wang TY, Cain CA, Fowlkes JB, Sapozhnikov OA, Bailey MR, Xu Z. Cavitation clouds created by shock scattering from bubbles during histotripsy. J Acoust Soc Am 2011;130:1888-1898.

6. Hall TL, Kieran K, Ives K, Fowlkes JB, Cain CA, Roberts WW. Histotripsy of rabbit renal tissue in vivo: temporal histologic trends. J Endourol 2007;21:1159-1166.

7. Hall T, Lee G, Hernandez L, Cain C. Relaxation properties of cavitation induced tissue lesions. In Proccedings of the 15th Annual Meeting of ISMRM, Berlin, Germany, 2007. Abstract 1118.

8. Allen SP, Roberts WW, Hall TL, Cain CA, Hernandez L. Characterization of in vivo histotripsy lesions using high field MRI. In Proceedings of the 20th Annual Meeting of ISMRM, Melbourne, Australia, 2012. Abstract 1582.

9. Kim Y, Fifer CG, Gelehrter SK, Owens GE, Berman DR, Vlaisavljevich E, Allen SP, Ladino-Torres MF, Xu Z. Developmental impact and lesion maturation of histotripsy-mediated non-invasive tissue ablation in a fetal sheep model. Ultrasound Med Biol 2013;39:1047-1055.

10. Allen SP, Hall TL, Cain CA, Hernandez L. MR-based targeting of histotripsy therapy at 7T. In Proceedings of the 21st Annual Meeting of ISMRM, Salt Lake City, Utah, USA, 2013. Abstract 1831.

11. Lin SC, Chen CW, Wu CH, Wang CH, Kang ST, Yeh CK, Chen WS, Peng HH. Real-time monitoring of intertial cavitation effect on diluted microbubbles by MRI. In Proceedings of the 21st Annual Meeting of ISMRM, Salt Lake City, Utah, 2013. Abstract 1825.

12. Alexander AL, Mccreery TT, Barrette TR, Gmitro AF, Unger EC. Microbubbles as novel pressure-sensitive MR contrast agents. Magn Reson Med 1996;35:801-806.

13. Cheung JS, Chow AM, Guo H, Wu EX. Microbubbles as a novel contrast agent for brain MRI. Neuroimage 2009;46:658-664. 
14. Dharmakumar R, Plewes DB, Wright GA. On the parameters affecting the sensitivity of MR measures of pressure with microbubbles. Magn Reson Med 2002;47:264-273.

15. Ueguchi T, Tanaka Y, Hamada S, Kawamoto R, Ogata Y, Matsumoto M, Nakamura H, Johkoh T. Air microbubbles as MR susceptibility contrast agent at 1.5 tesla. Magn Reson Med Sci 2006;5:147-150.

16. Wong KK, Huang I, Kim YR, Tang H, Yang ES, Kwong KK, Wu EX. In vivo study of microbubbles as an MR susceptibility contrast agent. Magn Reson Med 2004;52:445-452.

17. Hynynen K, Mcdannold N, Vykhodtseva N, Jolesz FA. Noninvasive MR imaging-guided focal opening of the blood-brain barrier in rabbits. Radiology 2001;220:640-646.

18. Hynynen K, Mcdannold N, Sheikov NA, Jolesz FA, Vykhodtseva N. Local and reversible blood-brain barrier disruption by noninvasive focused ultrasound at frequencies suitable for trans-skull sonications. Neuroimage 2005;24:12-20.

19. Hynynen K. MRIgHIFU: a tool for image-guided therapeutics. J Magn Reson Imaging 2011;34:482-493

20. Xu Z, Hall TL, Fowlkes JB, Cain CA. Optical and acoustic monitoring of bubble cloud dynamics at a tissue-fluid interface in ultrasound tissue erosion. J Acoust Soc Am 2007;121:2421-2430.

21. Xu Z, Raghavan M, Hall TL, Mycek MA, Fowlkes JB, Cain CA. Evolution of bubble clouds induced by pulsed cavitational ultrasound therapy - Histotripsy. IEEE Trans Ultrason Ferroelectr Freq Control 2008; 55:1122-1132.

22. Wang TY, Xu Z, Hall TL, Fowlkes JB, Cain CA. An efficient treatment strategy for histotripsy by removing cavitation memory. Ultrasound Med Biol 2012;38:753-766.

23. Stejskal EO, Tanner JE. Spin diffusion measurements: spin echoes in the presence of a time-dependent field gradient. J Chem Phys 1965; 42:288-292.
24. Le Bihan D, Breton E, Lallemand D, Grenier P, Cabanis E, LavalJeantet M. MR imaging of intravoxel incoherent motions: application to diffusion and perfusion in neurologic disorders. Radiology 1986; 161:401-407.

25. Muthupillai R, Rossman PJ, Lomas DJ, Greenleaf JF, Riederer SJ, Ehman RL. Magnetic resonance imaging of transverse acoustic strain waves. Magn Reson Med 1996;36:266-274.

26. Wu T, Felmlee JP, Greenleaf JF, Riederer SJ, Ehman RL. MR imaging of shear waves generated by focused ultrasound. Magn Reson Med 2000;43:111-115.

27. Sarvazyan AP, Rudenko OV, Swanson SD, Fowlkes JB, Emelianov SY. Shear wave elasticity imaging: a new ultrasonic technology of medical diagnostics. Ultrasound Med Biol 1998;24:1419-1435.

28. Mcdannold N, Maier SE. Magnetic resonance acoustic radiation force imaging. Med Phys 2008;35:3748-3758.

29. Radicke M, Engelbertz A, Habenstein B, Lewerenz M, Oehms O, Trautner P, Weber B, Wrede S, Maier K. New image contrast method in magnetic resonance imaging via ultrasound. Hyperfine Interact 2008;181:21-26.

30. Starritt HC, Hoad CL, Duck FA, Nassiri DK, Summers IR, Vennart W. Measurement of acoustic streaming using magnetic resonance. Ultrasound Med Biol 2000;26:321-333.

31. Sinnaeve D. The Stejskal-Tanner equation generalized for any gradient shape-an overview of most pulse sequences measuring free diffusion. Concepts Magn Reson 2012;40A:39-65.

32. Le Bihan D, Breton E, Lallemand D, Aubin ML, Vignaud J, LavalJeantet M. Separation of diffusion and perfusion in intravoxel incoherent motion MR imaging. Radiology 1988;168:497-505.

33. Parsons JE, Cain CA, Fowlkes JB. Cost-effective assembly of a basic fiber-optic hydrophone for measurement of high-amplitude therapeutic ultrasound fields. J Acoust Soc Am 2006;119:1432-1440. 